\title{
Experimental study: Effect of pore geometry and structural heterogeneity on the repetitive two-phase fluid flow in porous media and its implications to PM-CAES
}

\author{
Jingtao Zhang ${ }^{1}$, Haipeng Zhang ${ }^{2}$, Donghee Lee ${ }^{3}$, Sangjin $\mathrm{Ryu}^{4}$, and Seunghee Kim ${ }^{5,}$ \\ ${ }^{1}$ Dept. of Civil and Environmental Engineering, Univ. of Nebraska-Lincoln, Omaha, NE, USA \\ ${ }^{2}$ Dept. of Mechanical and Materials Engineering, Univ. of Nebraska-Lincoln, Lincoln, NE, USA \\ ${ }^{3}$ Dept. of Genetics, Cell Biology and Anatomy, Univ. of Nebraska Medical Center, Omaha, NE, USA \\ ${ }^{4}$ Dept. of Mechanical and Materials Engineering, Nebraska Center for Materials and Nanoscience, Univ. of Nebraska-Lincoln, Lincoln, \\ NE, USA \\ ${ }^{5}$ Dept. of Civil and Environmental Engineering, Nebraska Center for Materials and Nanoscience, Univ. of Nebraska-Lincoln, Omaha, \\ NE, USA
}

\begin{abstract}
Compressed air energy storage in porous media (PM-CAES) has recently been suggested as a promising alternative to existing CAES plants. PM-CAES incurs repetitive two-phase fluid flow caused by the cyclic injection and withdrawal of air to/from the porous medium that is initially saturated with the formation water during the operation. Therefore, predicting the overall macro-scale performance of porous media for energy storage requires a better understanding of repetitive two-phase fluid flow in the pore network at the fundamental pore-scale level. To answer this need, we conducted an experimental study using the microfluidics technology; we constructed polydimethylsiloxane (PDMS)-based micromodels with two different geometries (Type I: circular solids and Type II: square solids) and three different structural heterogeneities (coefficient of variation: $\mathrm{COV}=0,0.25$ and 0.5 ). Then, we applied a total of ten injectionwithdrawal cycles to each micromodel (i.e., ten cyclic drainage-imbibition processes) at different flow rate conditions $(Q=0.01$ and $0.1 \mathrm{ml} / \mathrm{min})$. It was observed that the displacement patterns of the initially residing fluid (wetting fluid; oil in this study) and the injected fluid (non-wetting fluid; water in this study) were greatly influenced by the geometry and heterogeneity of the pore structure, and imposed flow rate. Results such as the effective sweep efficiency and residual saturation of the non-wetting fluid were analyzed at each drainage-imbibition cycle to aid in understanding the impact of repetitive fluid flow. The experimental observations imply that the flow rate and structural heterogeneity may influence the efficiency of PM-CAES more than the pore geometry does.
\end{abstract}

\section{Introduction}

Compressed air energy storage (CAES) has been reemerging recently due to the large energy storage capacity it can provide, as well as other merits such as being low cost and potentially easy access to geologic formations for the storage [1]. To date, there are only two existing commercial CAES plants (290 MW - later upgraded to 321 MW CAES plant in Huntorf, Germany, and the 110 MW CAES plant in McIntosh, Alabama, USA), partly because of the lack of policy and economic drivers [2]. Those plants use salt caverns to store compressed air. On the other hand, underground porous rock formations (e.g., saline aquifers and depleted gas reservoirs) with impermeable upper and lower layers have been suggested as an alternative of compressed air storage medium, which is referred to as the porous media-CAES (PM-CAES; Figure 1). The consolidated and/or partially consolidated sandstones or carbonates with natural fractures are widely distributed in saline aquifers and hydrocarbon reservoirs. Compared to the salt cavern, those geologic formations show a much wider distribution and larger storage capacity, thereby loom a promising future application [3].

The working mechanism of PM-CAES includes the injection of compressed air using a motor and a compressor into an underground porous medium for storage, and its release back to a surface unit to regenerate electricity by driving an air turbine and a generator (Figure 1). In general, the air storage in porous aquifer exhibits a constant volume configuration with an air pressure variation during the operation. The minimum storage pressure is determined by the required inlet pressure to the air turbine, while the maximum storage pressure is limited by the hydrostatic pressure at the top of the aquifer. When the air pressure in the aquifer declines during the air withdrawal, the movement of the air-water interface driven by the pressure gradient is unavoidable, particularly for the porous aquifer with high permeability [4-6]. That

* Corresponding author: seunghee.kim@unl.edu 
is, a transition zone between the air storage and water saturation could exist [4], in which the two-phase immiscible fluid flow (i.e., drainage process: non-wetting air displaces wetting water, and imbibition process: wetting water reoccupies the pore space) will be repeated during the air injection and withdrawal. In this respect, a good understanding of the repetitive two-phase fluid flow in porous media at the fundamental pore-scale level is critically important for the reliable prediction of the overall macro-scale performance of PM-CAES. In this study, we aim to explore the effect of structural heterogeneity and pore geometry that characterize the geologic formation and the effect of flow rate to advance our understanding of the repetitive two-phase fluid flow at the pore-scale.

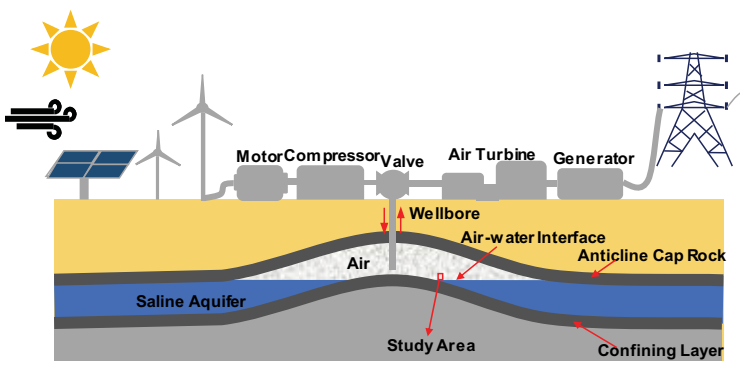

Figure 1. A schematic illustration of PM-CAES.

\section{Governing numbers}

The two-phase fluid flow in porous media is heavily influenced by the capillary pressure $\left(P_{c}\right)$, capillary number $(C a)$, and mobility ratio $(M)[7,8]$. Their mathematical expressions are as follows:

$$
P_{c}=\frac{2 \gamma \cos \theta}{r}, C a=\frac{\mathrm{v} \cdot \mu_{n w}}{\gamma \cos \theta}, \text { and } M=\frac{\mu_{n w}}{\mu_{w}}
$$

where $\gamma$ is the interfacial surface tension coefficient $[\mathrm{mN} / \mathrm{m}], \theta$ is the contact angle of a solid-fluid-fluid system [deg], $r$ is the radius of a flow channel, $V$ is the velocity of an advancing non-wetting fluid $[\mathrm{m} / \mathrm{s}], \mu_{n w}$ is the viscosity of a non-wetting fluid $[\mathrm{Pa} \cdot \mathrm{s}]$, and $\mu_{w}$ is the viscosity of a wetting fluid [Pa.s].

\section{Experimental study}

\subsection{Pore structure of the micromodel}

Structurally heterogeneous pore-network models - Type I with circular solids, and Type II with square solids - were designed in reference to the geometry and dimensions of the homogeneous micromodels used in Zhang et al. [9] to emulate the consolidated and/or partially consolidated sandstone reservoir and hydrocarbon reservoir with naturally fractured carbonates, respectively. The dimension of the main domain was $20 \mathrm{~mm} \times 20 \mathrm{~mm}$, and there were $50 \times 48$ (parallel $\times$ perpendicular to the pressure gradient) solids in the main domain of pore-network Type I and II (Figure 2). This dimension is larger than the representative elementary volume (REV) of sandstones and carbonates, respectively. Thus, observations made from the small-scale fluid flow test in this study can be used for predicting the macro-scale flow phenomena in the field [9].

The diameter of all circular solids was the same at 360 $\mu \mathrm{m}$ in Type I, and the width of all square solids in Type II was $320 \mu \mathrm{m}$. The code was written using MATLAB to determine the location of each solid in a way that the width of pore throats followed the log-normal distribution with the mean value of $40 \mu \mathrm{m}$ in Type I and $80 \mu \mathrm{m}$ in Type II for both vertical and horizontal directions. These mean values are also the widths of pore throats in the homogeneous reference micromodels [9], except the upper and lower edges where the width of pore throats parallel to the pressure gradient is half of the corresponding mean values $(20 \mu \mathrm{m}$ in Type I and $40 \mu \mathrm{m}$ in Type II). The coefficient of variation (COV) of the log-normal distribution was either 0.25 or 0.5 in this study. The minimum width of the pore throat was set as $20 \mu \mathrm{m}$ due to the resolution limit of the fabrication method. The height of all pore throats was identical at $100 \mu \mathrm{m}$. Once the coordinates of all solids were determined using the code, the information was used for reproducing the pore structure in AutoCAD (Autodesk).

In this study, two independently designed pore structures for each $\mathrm{COV}=0,0.25$, and 0.5 were used, respectively. One representative pore structure for each $\mathrm{COV}$ is shown in Figure 2. We confirmed that the porosity of all fabricated Type I and II micromodel devices was 0.36 and $0.36-0.38$, respectively, and their permeability was also very similar (in the rage of $\sim 4.6$ Darcy to $\sim 7.5$ Darcy in Type I and $~ 4.0$ Darcy to $\sim 6.4$ Darcy in Type II). Both porosity and permeability satisfy the requirements for being a viable storage medium of compressed air [5]. Particularly, the permeability values in this study are higher than 1 Darcy, which means "exceptional" in the permeability classification and can significantly facilitate the movement of the air-water interface.

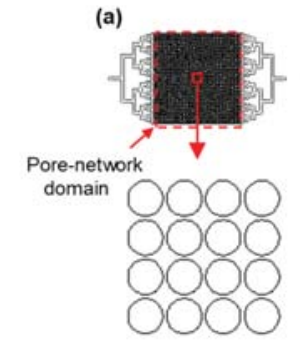

$\operatorname{cov}=0$

(b)

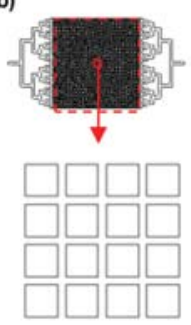

$\operatorname{cov}=0$

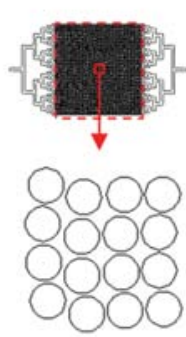

$\operatorname{cov}=0.25$

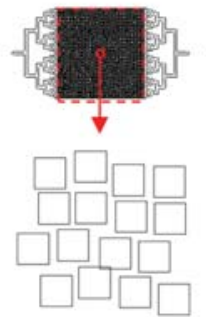

$\mathrm{cov}=0.25$

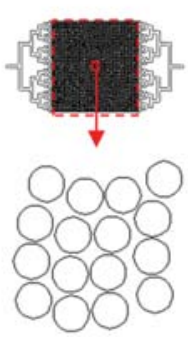

$\mathrm{cov}=0.5$

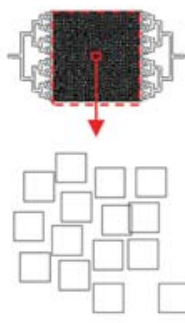

cov $=0.5$
Figure 2. Microfluidic pore-network models with different $\mathrm{COV}$ : (a) Type I and (b) Type II. 


\subsection{Fabrication of the pore-network micromodel}

We used a standard microfluidics fabrication technique using polydimethylsiloxane (PDMS) to make the porenetwork micromodels. It contained three parts; the inlet, the main pore-network domain, and the outlet (Figure 2). The inlet and outlet were designed in a tree-like shape. A transparent photomask $(254,000 \mathrm{dpi})$ was then prepared based on the design and used to produce a master mold using photolithography. The master mold consisted of the positive relief of SU-8 100 photoresist (MicroChem) on a silicon wafer. After that, PDMS was prepared, poured onto the mold, and cured in an oven at $65^{\circ} \mathrm{C}$ for 6 hours. The cured PDMS was then peeled off from the mold, punched for the inlet and outlet, and bonded to a glass slide (that was spin-coated with a thin layer of cured PDMS in advance) via plasma activation. Then, the fabricated device was cured in the oven at $65^{\circ} \mathrm{C}$ for one day. Finally, the inlet and outlet holes were inserted with a $2.5 \mathrm{~cm}$-long tube (Tygon tubing, $0.508 \mathrm{~mm}[0.02$ "] ID $\times 1.524 \mathrm{~mm}$ $\left[0.06^{\prime \prime}\right]$ OD). It marks the completion of a hydrophobic (or oleophilic) PDMS micromodel fabrication.

\subsection{Materials}

Mineral oil (Fisher Chemical BP2629-1) and water were selected as the wetting fluid $\left(\mu_{w}=4.2 \times 10^{-2} \mathrm{~Pa} \cdot \mathrm{s}\right.$, and $\rho_{w}=$ $820-880 \mathrm{~kg} / \mathrm{m}^{3}$ at $25^{\circ} \mathrm{C}$ ) [10] and the non-wetting fluid (colored for visualization; $\mu_{n w}=1.0 \times 10^{-3} \mathrm{~Pa} \cdot \mathrm{s}, \rho_{n w}=1,000$ $\mathrm{kg} / \mathrm{m}^{3}$ ), respectively, because PDMS is oleophilic. The surface tension coefficient between water and mineral oil is about $52 \mathrm{mN} / \mathrm{m}$ at $25^{\circ} \mathrm{C}$ [11], and we assumed $\theta=45^{\circ}$ for simplicity [9]. With that fluid selection and imposed flow rate, $\mathrm{Ca}$ is closer to the lower range of potential CAES projects, and $M$ is very similar to that of the air/water condition. Thus, the choice of wetting and nonwetting fluids and PDMS solid retains intimate relevancy to the two-phase fluid flow situation of an actual PMCAES operation.

\subsection{Experimental setup and procedure}

The newly fabricated micromodel was pre-saturated with the oil before each test. A syringe (BD, $3 \mathrm{ml}$ ) and a highprecision syringe pump (PHD ULTRA) were used to impose a constant flow rate $(\mathrm{Q}=0.01 \mathrm{ml} / \mathrm{min}$ or 0.1 $\mathrm{ml} / \mathrm{min}$ ) during the total of ten repetitive drainage and imbibition cycles. An empty container was placed at the outlet side to collect the effluent fluid. The injection duration during both drainage and imbibition processes was set at 330 seconds for $\mathrm{Q}=0.01 \mathrm{ml} / \mathrm{min}$ and 33 seconds for $\mathrm{Q}=0.1 \mathrm{ml} / \mathrm{min}$ so that 2.5 pore volumes of either nonwetting or wetting fluid was supplied to the micromodel. The fluid flow in the main domain of the micromodel was captured using a microscope (LEICA S90), combined with a high-speed camera (LEICA MIC 190 HD).

Drainage was initiated from the right-hand side of the micromodel using the syringe pump. Drainage was continued for the injection duration, and then the pump was stopped, and the two valves were closed immediately: completion of the $1^{\text {st }}$ drainage step (from right- to the lefthand side). Next, outer tubes were replaced with new ones filled with either mineral oil (left side) or water (right side), and the syringe pump was moved to the left, along with a new syringe filled with oil. A new empty container was also placed on the right-hand side. Afterward, two valves were opened again, the syringe pump started, and oil began to reoccupy the micromodel. Imbibition was continued for another injection duration. Then, the two valves were closed in the same way: completion of the $1^{\text {st }}$ imbibition step (from left- to the right-hand side). This procedure was repeated to complete a total of ten cycles.

These repetitive drainage-imbibition tests were conducted for two implementations with a homogeneous micromodel $(\mathrm{COV}=0)$, newly fabricated each time, and one implementation with each of the four heterogeneous micromodels (two micromodels with $\mathrm{COV}=0.25$, and the other two with COV $=0.5$ ) of Type I and II, respectively. The Reynolds number of the fluid flow in the tree-like inlet of Type I and II was calculated as $R e=0.5-5$ and $0.01-0.1$ for the drainage and imbibition, respectively. Therefore, all flow experiments in this study belonged to the laminar flow regime.

\section{Experimental results and analyses}

\subsection{Drainage and imbibition}
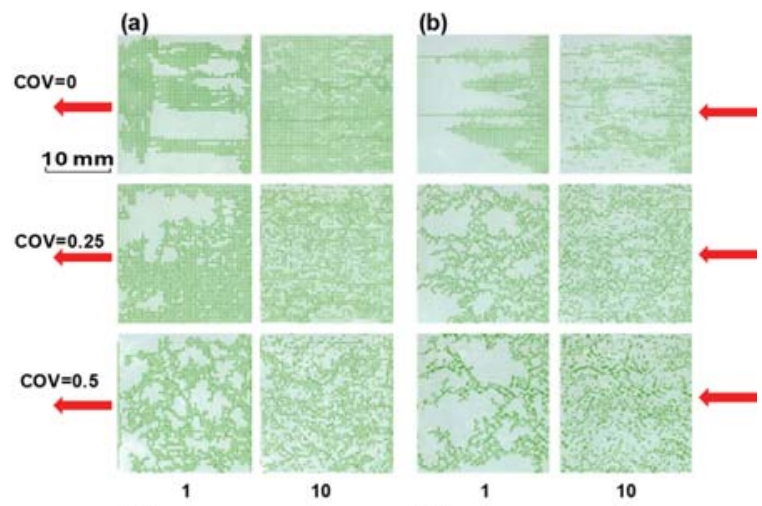

(c)
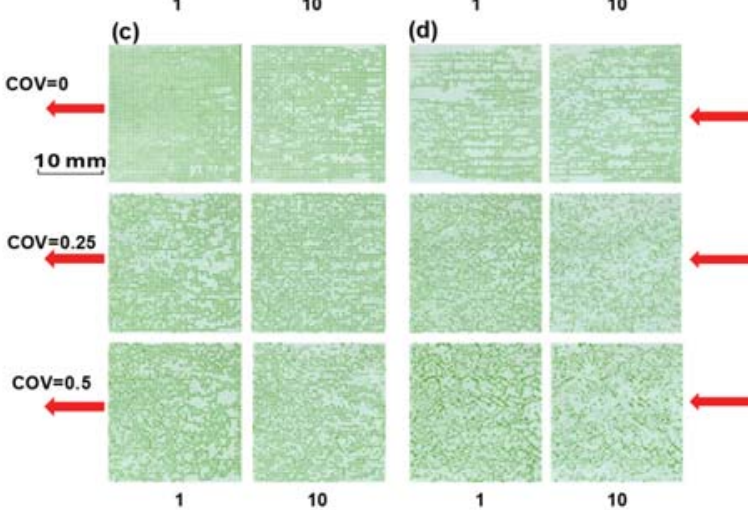

Figure 3. Distribution of water (the green colored non-wetting fluid) at the end of $1^{\text {st }}$ and $10^{\text {th }}$ drainage steps in (a) Type I and (b) Type II at $0.01 \mathrm{ml} / \mathrm{min}$, and (c) Type I and (d) Type II at 0.1 $\mathrm{ml} / \mathrm{min}$ (the number below each column of images indicates the turn of cycles). Red arrows: the direction of water injection. 
The distributions of water (green-colored; non-wetting) at the end of $1^{\text {st }}$ and $10^{\text {th }}$ drainage step from one representative test at each $\operatorname{COV}(\mathrm{COV}=0,0.25$, and 0.5$)$ at two different flow rates $(Q=0.01$ and $0.1 \mathrm{ml} / \mathrm{min})$ for Type I and II are compiled in Figure 3. It was observed that the invasion of water in Type I was more branched as COV increased (i.e., more heterogeneous) at the end of the first drainage, which implies the stronger influence of capillary pressures. Such capillary fingering was more conspicuous under the lower flow rate $(0.01 \mathrm{ml} / \mathrm{min}$; compare Figure $3-\mathrm{a}$ and $\mathrm{c}$ ). It was also found that the distribution of water became more uniform as the repetitive drainage-imbibition continued (compare the $10^{\text {th }}$ drainage with the $1^{\text {st }}$ drainage step). This trend was more substantial at the lower flow rate.

On the other hand, the invasion of water in Type II was prevalent in the direction of the pressure gradient when $\mathrm{COV}=0$, particularly at the higher flow rate. The invasion of water became more localized and branched as the heterogeneity level increased, which shows the influence of capillarity again. Similar to Type I, the distribution of water became more uniform as the repetitive injection cycle continued at the lower flow rate, but the effect of cyclic flow was less obvious in the high flow rate.
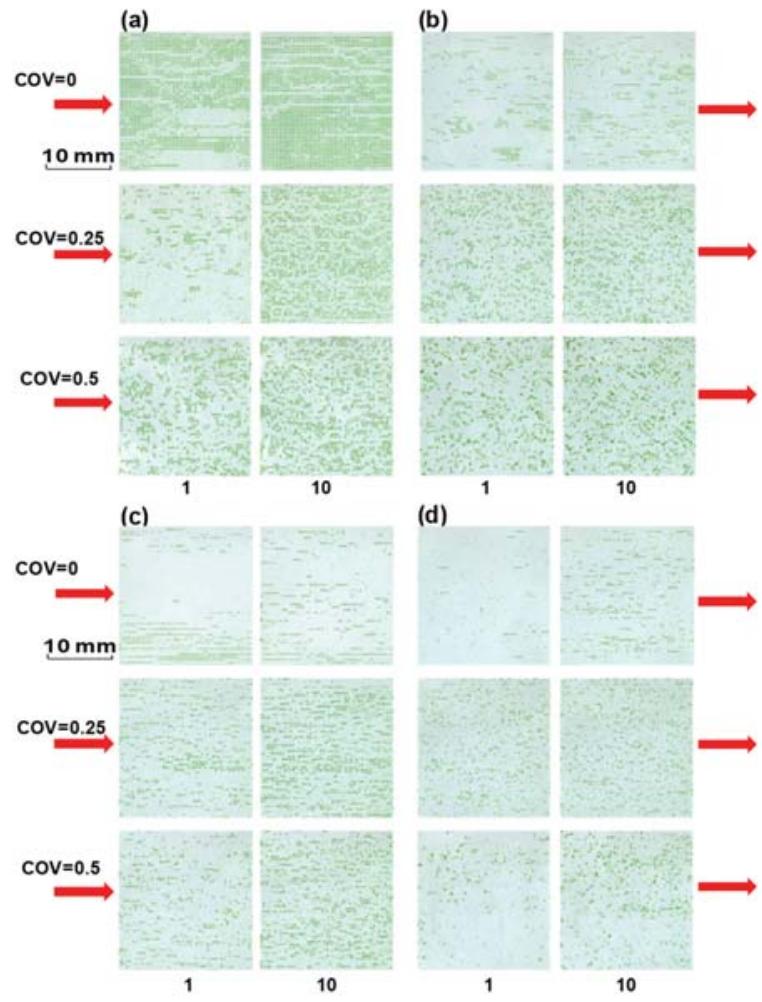

Figure 4. Distribution of water (the green non-wetting fluid) at the end of $1^{\text {st }}$ and $10^{\text {th }}$ imbibition step in (a) Type I and (b) Type II at $0.01 \mathrm{ml} / \mathrm{min}$, and (c) Type I and (d) Type II at $0.1 \mathrm{ml} / \mathrm{min}$ (the number below each column of images indicates the turn of cycles). Red arrows: the direction of oil injection.

The distributions of residual water (green-colored) at the end of the $1^{\text {st }}$ and $10^{\text {th }}$ imbibition step from one representative test at each COV are compiled in Figure 4. It was observed that the distribution of residual water was predominantly aligned in parallel with the pressure gradient direction (i.e., horizontal) in Type I homogeneous micromodel $(\mathrm{COV}=0)$ at both flow rates. That distribution became more dispersed as the structural heterogeneity increased (COV $\rightarrow 0.5$ ). It was clearly observed that less water remained in the main domain of pore-network as $Q$ increased (compare Figure 4-a and c). Unlike Type I, the distribution of water in Type II homogeneous micromodels was more sporadic and random. Besides, it seems that more water was left in the main domain of pore space as the COV increased at both flow rates. Similar to Type I, the higher flow rate in Type II helped to displace more water by oil (compare Figure 4-b and d). Lastly, Type II was observed to be more efficient than Type I for the water withdrawal (that is, withdrawal of air in a real PM-CAES) under the given flow rates (compare Figure 4$a$ and $b$, and 5-c and d).

\subsection{Injection efficiency}

We used the effective sweep efficiency in this study for the quantitative evaluation of the injection efficiency of the non-wetting fluid. Effective sweep efficiency, $e E_{\mathrm{nw}}$, is defined as the ratio of the difference of the pore volume occupied by the non-wetting fluid after $\left(V_{\mathrm{nw}}\right)$ and before $\left(V_{\text {rnw }}\right)$ drainage to the pore volume occupied by the wetting fluid $\left(V_{\mathrm{w}}\right)$ before the drainage; i.e., $e E_{\mathrm{nw}}=\left(V_{\mathrm{nw}}-V_{\mathrm{rnw}}\right) / V_{\mathrm{w}}$. A higher effective sweep efficiency implies that water can occupy more pore space during each drainage process, and it is beneficial to the actual operation of PM-CAES. By analogy, injected air can occupy more pore space in a targeted porous medium.
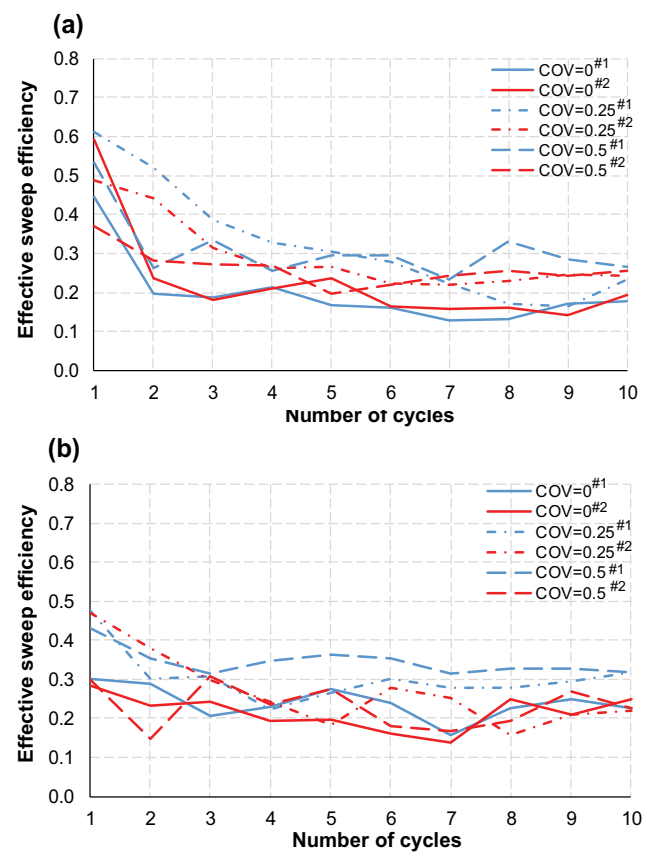

Figure 5. Effective sweep efficiency of water, $e E_{n w}$, at $Q=0.01$ $\mathrm{ml} / \mathrm{min}$ for (a) Type I and (b) Type II micromodels.

The effective sweep efficiency $\left(e E_{\mathrm{nw}}\right)$ shows that the higher flow rate indeed improved the injection efficiency of the non-wetting fluid for the same pore structure 
(compare Figures 5 and 6). It is noticeable that $e E_{\mathrm{nw}}$ was lowest for $\mathrm{COV}=0$ at the lower flow rate $(Q=0.01 \mathrm{ml} / \mathrm{min})$ but the highest at the higher flow rate $(Q=0.1 \mathrm{ml} / \mathrm{min})$ in both Types I and II. In detail, when $Q=0.01 \mathrm{ml} / \mathrm{min}, e E_{\mathrm{nw}}$ converged between 0.15 and 0.25 after the first cycle in both Types I and II homogeneous micromodels, whereas, $e E_{\mathrm{nw}}$ of heterogeneous micromodels fluctuated between 0.15 and 0.35 (in overall, higher for $\mathrm{COV}=0.5$ than 0.25 ) in both Types I and II. It implies that it was relatively easier for water to displace oil in heterogeneous micromodels when the capillarity was still prevalent.

In contrast, when $Q=0.1 \mathrm{ml} / \mathrm{min}, e E_{\mathrm{nw}}$ of the homogeneous micromodels hovered around $0.4-0.45$ in Type I and $0.35-0.45$ in Type II, and that of heterogeneous micromodels fluctuated between 0.3 and 0.45 (lower for $\mathrm{COV}=0.5$ than 0.25 ) in Type I, and between 0.3 and 0.4 in Type II. It suggests that the occupational efficiency of the non-wetting fluid might be better in the homogeneous pore geometry when the flow rate is high enough so that the capillary pressure no longer prevailed in either Type I or II micromodels. By the way, the influence of different pore geometry (Type I and II) was not significant on the effective sweep efficiency for the same COV and flow rate (compare Figures 5-a and b, and Figures 6-a and b).
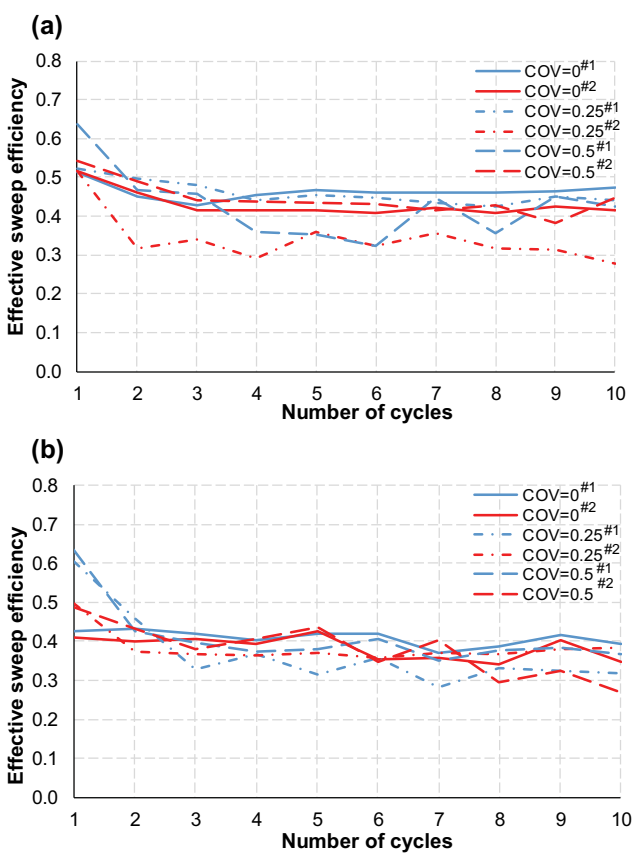

Figure 6. Effective sweep efficiency of water, $e E_{n w}$, at $Q=0.1$ $\mathrm{ml} / \mathrm{min}$ for (a) Type I and (b) Type II micromodels.

\subsection{Withdrawal efficiency}

The effective residual saturation in this study was used for the quantitative evaluation of the withdrawal efficiency of the non-wetting fluid. The effective residual saturation, $e S_{\text {rnw }}$, is expressed as the ratio of the pore volume still occupied by residual non-wetting fluid $\left(V_{\text {rnw }}\right)$ to the pore volume of non-wetting fluid $\left(V_{\mathrm{nw}}\right)$ before the imbibition; i.e., $e S_{\text {rnw }}=V_{\text {rnw }} / V_{\text {nw }}$. Lower $e S_{\text {rnw }}$ implies more oil can reoccupy the pore space, and thus, more water can be removed during each imbibition process. However, too low $e S_{\text {rnw }}$ may be detrimental to the actual operation of PM-CAES, for water in the actual porous formations (wetting fluid) can come back closer to the wellbore during the air withdrawal that can cause the water coning problem.

It was observed that $e S_{\text {rnw }}$ was highest in Type I but lowest in Type II homogeneous models $(\mathrm{COV}=0)$ when $Q=0.01 \mathrm{ml} / \mathrm{min}$ (Figure $7-\mathrm{a}$ and $\mathrm{b}$ ). It implies that more non-wetting fluid would remain trapped in Type I homogeneous pore-network during the imbibition at low $Q$, showing a substantial influence of capillarity; whereas, the straight pore throat with a low aspect ratio (the ratio of the width of the pore space to the width of pore throat) in Type II homogeneous pore-network dwarfed the capillary effect and facilitated the reoccupation of oil at low $Q$. However, when the structural heterogeneity increased, the aspect ratio of Type II became less influential (that is, higher residual water saturation with higher COV).

On the other hand, $e S_{\text {rnw }}$ was lowest in both Types I and II homogeneous models when $Q$ was increased (Figure 8-a and b). It implies that $Q$ was sufficient to overcome most of the capillary pressures in both geometries of homogeneous pore-network, while its dominance diminished in the heterogeneous pore-network $(\mathrm{COV}=0.25$ and 0.5$)$ as a result of the distribution of wider size range of pore throats. These results suggest that the withdrawal efficiency of the non-wetting fluid would be better in the homogeneous pore structure of both Types I and II when the flow rate is high enough so that the capillary pressure no longer prevails. Besides, the higher flow rate can yield an improved withdrawal efficiency for the same pore structure, as long as the water coning problem does not occur (compare Figures $7 \mathrm{a}$ and $8 \mathrm{a}$, and compare Figures $7 b$ and $8 b$ ).

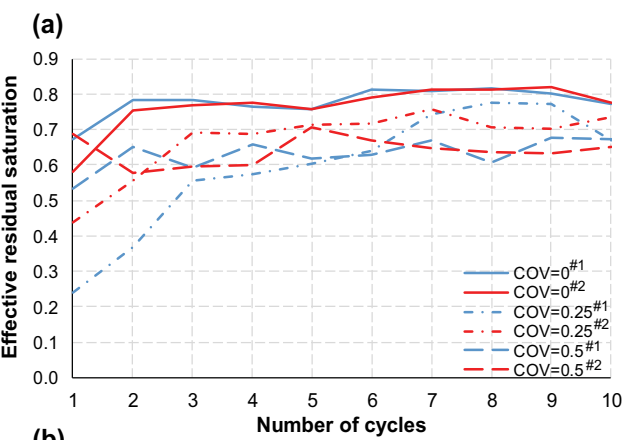

(b)

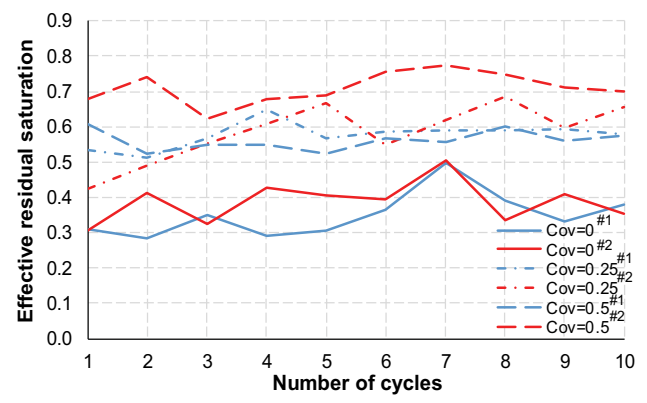

Figure 7. Effective residual water saturation, $e S_{r n w}$, at $Q=0.01$ $\mathrm{ml} / \mathrm{min}$ for (a) Type I and (b) Type II micromodels. 
(a)

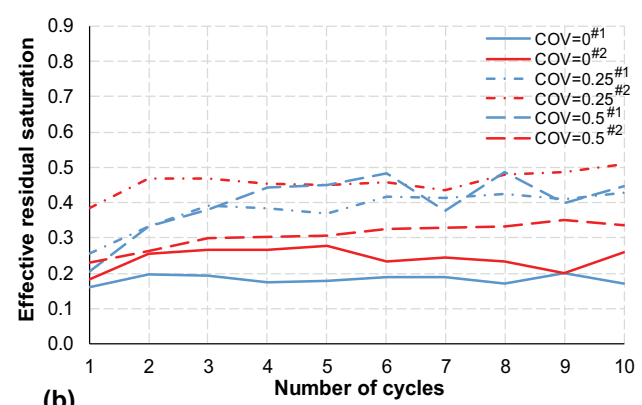

(b)

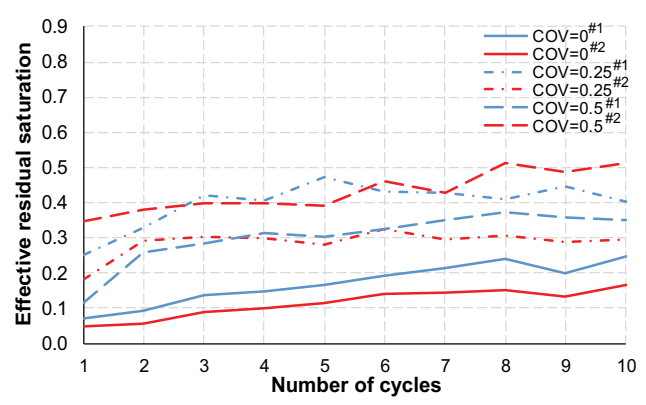

Figure 8. Effective residual water saturation, $e S_{r n w}$, at $Q=0.1$ $\mathrm{ml} / \mathrm{min}$ for (a) Type I and (b) Type II micromodels.

\section{Conclusions}

We fabricated Type I (to emulate consolidated and/or partially consolidated sandstones) and Type II (to emulate carbonates with natural fracture networks) PDMS-based micromodels with different structural heterogeneity (COV $=0,0.25$, and 0.5 ) and conducted the repetitive two-phase fluid flow tests with them at two different flow rates $(Q=$ 0.01 and $0.1 \mathrm{ml} / \mathrm{min}$ ). We then analyzed the flow morphology and injection/withdrawal efficiencies. Salient observations are summarized below.

- The occupation of the non-wetting fluid at the end of the first drainage in Type I was more branched as the structural heterogeneity intensified at all flow rates, which implies the influence of capillary pressures. On the other hand, the invasion of water in Type II homogeneous micromodels was aligned preferentially in parallel with the direction of pressure gradient, and more straight flow paths were observed as the flow rate increased. This flow pattern turned more localized with the increased structural heterogeneity at both flow rates.

- During imbibition, the distribution of the residual non-wetting fluid was aligned more parallel with the direction of the pressure gradient in Type I homogeneous micromodel, and less non-wetting fluid remained in the micromodel as the flow rate increased. On the other hand, the distribution of the residual nonwetting fluid in all Type II micromodels exhibited sporadic and random characteristics. Similar to Type I, the higher flow rate led to the reoccupation of more wetting fluid in the pore space of Type II. Overall, less water was left behind in Type II.

- The effective sweep efficiency $\left(e E_{\mathrm{nw}}\right)$ was substantially affected by both flow rate and structural heterogeneity, but the effect of pore geometry was not significant. At the lower flow rate, $e E_{\mathrm{nw}}$ was higher as the heterogeneity increased, but the trend was opposite at the higher flow rate, implying that the homogeneous pore structure could accommodate more non-wetting fluid when the capillary effect diminished at the higher flow rate in both Types I and II geometries.

- The effective residual saturation $\left(e S_{\text {rnw }}\right)$ of the non-wetting fluid was largest in Type I but smallest in Type II homogeneous pore structure at the lower flow rate, respectively. It shows the substantial influence of the capillary pressure, which acted to trap the non-wetting fluid more in Type I homogeneous pore structure.

However, the straight pore throat with a small aspect ratio in Type II homogeneous micromodel dwarfed the influence of capillary pressure, stimulating the more displacement of the non-wetting fluid. The higher flow rate could help overcome this capillary effect in Type I homogeneous pore structure, which facilitated the displacement of the non-wetting fluid more evenly, resulting in the lowest $e S_{\text {rnw }}$. Besides, the higher flow rate can yield an improved withdrawal efficiency for the same pore structure, as long as the water coning problem does not occur.

Overall, the consolidated and/or partially consolidated sandstones (Type I) and naturally fractured carbonates (Type II) with a similar level of structural heterogeneity may yield a similar performance during the actual operation of PM-CAES at a high flow rate. On the other hand, it seems that Type II geometry may be better for the actual PM-CAES at a low flow rate - a similar injection efficiency is expected for both types, but more nonwetting fluid can be withdrawn in Type II geometry for power regeneration under the same imposed flow rate.

This research was supported by the Research Council: Interdisciplinary Grant at University of Nebraska-Lincoln for S. Kim and S. Ryu, the Start-up Grant for S. Kim at University of Nebraska-Lincoln, and American Chemical Society Petroleum 
Research Fund for S. Ryu. We appreciate Stephen Morin for his assist in microfluidics master mold fabrication.

\section{References}

[1] A. Rogers, A. Henderson, X. Wang, M. Negnevitsky, Compressed air energy storage: thermodynamic and economic review, in: IEEE Power Energy Soc. Gen. Meet., (2014).

[2] F. Crotogino, K.-U. Mohmeyer, R. Scharf, Huntorf CAES: more than 20 years of successful operation, in: Solut. Min. Res. Inst. Spring Meet., Orlando, Florida, USA, (2001).

[3] B. Wang, S. Bauer, Compressed air energy storage in porous formations: a feasibility and deliverability study, Pet. Geosci. 23(3), 306-314 (2017).

[4] L.E. Wiles, R.A. McCann, Water coning in porous media reservoirs for compressed air energy storage (No.PNL-3470), Battelle Pacific Northwest Labs, Richland, WA, USA, (1981).

[5] R.D. Allen, T.J. Doherty, R.L. Erikson, L.E. Wiles, Factors affecting storage of compressed air in porous rock reservoirs (No.PNL-4707), Battelle Pacific Northwest Labs, Richland, WA, USA, (1983).

[6] M. Medeiros, B. Robert, J. Fairchild, D. Imperato, C. Stinson, M. Ausburn, M. Tietze, S. Irani, A. Burzlaff, H. Moore, J. Day, B. Jordan, T. Holsey, D. Davy, K. Plourde, Technical feasibility of compressed air energy storage using a porous rock reservoir (CEC500-2018-029), California Energy Commission, (2018).

[7] R. Lenormand, E. Touboul, C. Zarcone, Numerical models and experiments on immiscible displacements in porous media, J. Fluid Mech. 189, 165-187 (1988).

[8] D.G. Avraam, A.C. Payatakes, Flow regimes and relative permeabilities during steady-state two-phase flow in porous media, J. Fluid Mech. 293, 207-236 (1995).

[9] J. Zhang, H. Zhang, D. Lee, S. Ryu, S. Kim, Microfluidic study on the two-phase fluid flow in porous media during repetitive drainage-imbibition cycles and implications to the CAES operation, Transp. Porous Media. 131, 449-472 (2020).

[10]https://lakeland.edu/PDFs/MSDS/1117/Mineral Oil (Fisher).pdf.

[11] S.M.S. Murshed, S.H. Tan, N.T. Nguyen, Temperature dependence of interfacial properties and viscosity of nanofluids for droplet-based microfluidics, J. Phys. D. Appl. Phys. 41(8) (2008). 